\title{
Observations of Mkn 421 in 2004 with HESS at large zenith angles
}

\author{
F. Aharonian ${ }^{1}$, A. G. Akhperjanian ${ }^{2}$, K.-M. Aye ${ }^{3}$, A. R. Bazer-Bachi ${ }^{4}$, M. Beilicke $^{5}$, W. Benbow ${ }^{1}$, D. Berge ${ }^{1}$,
} P. Berghaus ${ }^{6, \star}$, K. Bernlöhr ${ }^{1,7}$, C. Boisson ${ }^{8}$, O. Bolz ${ }^{1}$, I. Braun ${ }^{1}$, F. Breitling ${ }^{7}$, A. M. Brown ${ }^{3}$, J. Bussons Gordo ${ }^{9}$, P. M. Chadwick ${ }^{3}$, L.-M. Chounet ${ }^{10}$, R. Cornils ${ }^{5}$, L. Costamante ${ }^{1,20}$, B. Degrange ${ }^{10}$, A. Djannati-Atai ${ }^{6}$, L. O'C. Drury ${ }^{11}$, G. Dubus ${ }^{10}$, D. Emmanoulopoulos ${ }^{12}$, P. Espigat ${ }^{6}$, F. Feinstein ${ }^{9}$, P. Fleury ${ }^{10}$, G. Fontaine ${ }^{10}$, Y. Fuchs ${ }^{13}$, S. Funk ${ }^{1}$, Y. A. Gallant ${ }^{9}$, B. Giebels ${ }^{10}$, S. Gillessen ${ }^{1}$, J. F. Glicenstein ${ }^{14}$, P. Goret ${ }^{14}$, C. Hadjichristidis ${ }^{3}$, M. Hauser ${ }^{12}$, G. Heinzelmann ${ }^{5}$, G. Henri ${ }^{13}$, G. Hermann ${ }^{1}$, J. A. Hinton ${ }^{1}$, W. Hofmann ${ }^{1}$, M. Holleran ${ }^{15}$, D. Horns ${ }^{1}$, O. C. de Jager ${ }^{15}$, B. Khélifi ${ }^{1}$, Nu. Komin ${ }^{7}$, A. Konopelko ${ }^{1,7}$, I. J. Latham ${ }^{3}$, R. Le Gallou ${ }^{3}$, A. Lemière 6 ,

M. Lemoine ${ }^{10}$, N. Leroy ${ }^{10}$, T. Lohse ${ }^{7}$, A. Marcowith ${ }^{4}$, C. Masterson ${ }^{1,20}$, T. J. L. McComb ${ }^{3}$, M. de Naurois ${ }^{16}$,

S. J. Nolan ${ }^{3}$, A. Noutsos 3 , K. J. Orford ${ }^{3}$, J. L. Osborne ${ }^{3}$, M. Ouchrif ${ }^{16,20}$, M. Panter ${ }^{1}$, G. Pelletier ${ }^{13}$, S. Pita ${ }^{6}$,

G. Pühlhofer ${ }^{1,12}$, M. Punch ${ }^{6}$, B. C. Raubenheimer ${ }^{15}$, M. Raue ${ }^{5}$, J. Raux ${ }^{16}$, S. M. Rayner ${ }^{3}$, I. Redondo ${ }^{10,20, \star \star}$, A. Reimer ${ }^{17}$, O. Reimer ${ }^{17}$, J. Ripken ${ }^{5}$, L. Rob ${ }^{18}$, L. Rolland ${ }^{16}$, G. Rowell ${ }^{1}$, V. Sahakian ${ }^{2}$, L. Saugé ${ }^{13}$, S. Schlenker ${ }^{7}$, R. Schlickeiser ${ }^{17}$, C. Schuster ${ }^{17}$, U. Schwanke ${ }^{7}$, M. Siewert ${ }^{17}$, H. Sol ${ }^{8}$, R. Steenkamp ${ }^{19}$, C. Stegmann ${ }^{7}$, J.-P. Tavernet ${ }^{16}$, R. Terrier ${ }^{6}$, C. G. Théoret ${ }^{6}$, M. Tluczykont ${ }^{10,20}$, G. Vasileiadis ${ }^{9}$, C. Venter ${ }^{15}$, P. Vincent ${ }^{16}$, H. J. Völk ${ }^{1}$, and S. J. Wagner ${ }^{12}$

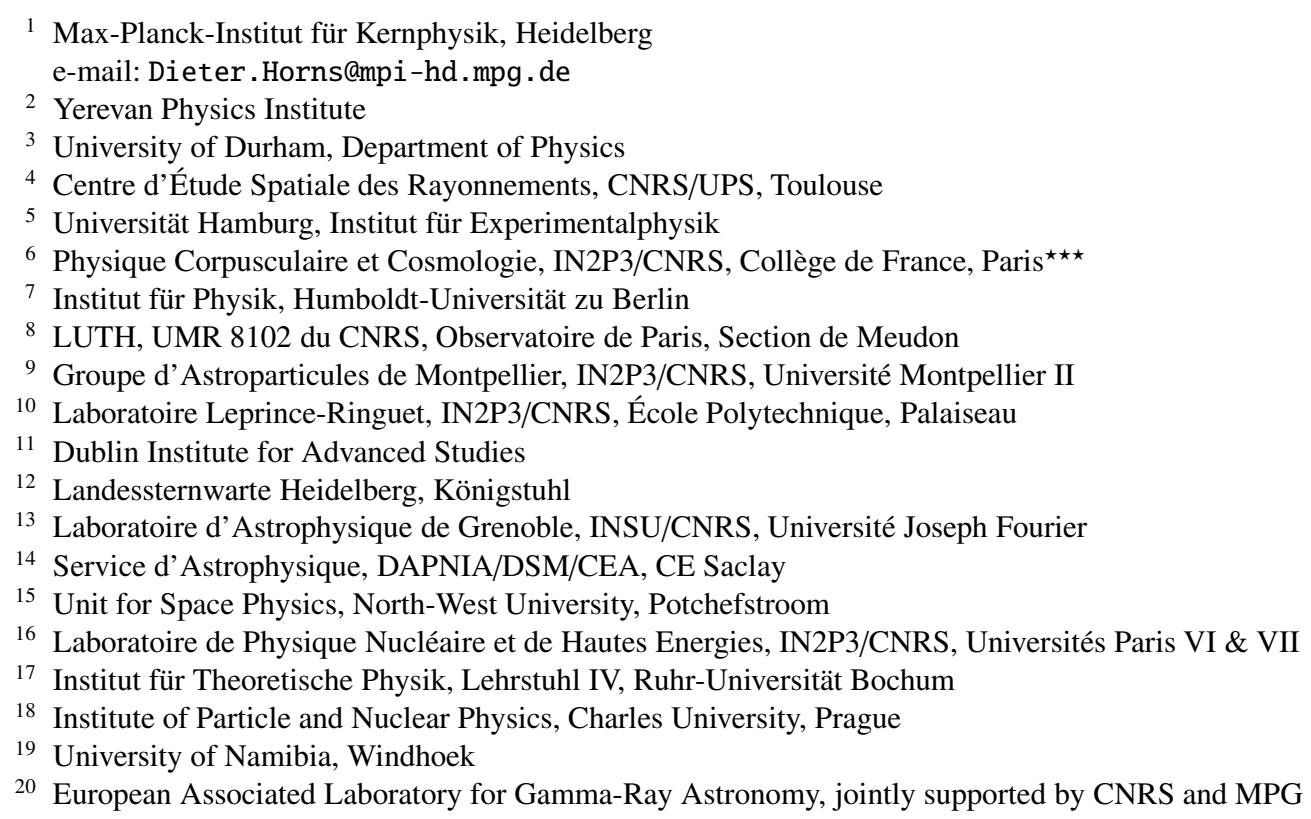

Received 11 March 2005 / Accepted 14 April 2005

\footnotetext{
* Université Libre de Bruxelles, Faculté des Sciences, Campus de la Plaine, CP230, Boulevard du Triomphe, 1050 Bruxelles, Belgium.

$\star \star$ Now at Department of Physics and Astronomy, Univ. of Sheffield, The Hicks Building, Hounsfield Road, Sheffield S3 7RH, UK.

$\star \star \star$ UMR 7164 (CNRS, Université Paris VII, CEA, Observatoire de Paris).
} 
Abstract. Mkn 421 was observed during a high flux state for nine nights in April and May 2004 with the fully operational High Energy Stereoscopic System (HESS) in Namibia. The observations were carried out at zenith angles of $60^{\circ}-65^{\circ}$, which result in an average energy threshold of $1.5 \mathrm{TeV}$ and a collection area reaching $2 \mathrm{~km}^{2}$ at $10 \mathrm{TeV}$. Roughly 7000 photons from Mkn 421 were accumulated with an average gamma-ray rate of 8 photons/min. The overall significance of the detection exceeds 100 standard deviations. The light-curve of integrated fluxes above $2 \mathrm{TeV}$ shows changes of the diurnal flux up to a factor of 4.3. For nights of high flux, intra-night variability is detected with a decay time of less than $1 \mathrm{~h}$. The time averaged energy spectrum is curved and is well described by a power-law with a photon index $\Gamma=2.1 \pm 0.1_{\text {stat }} \pm 0.3_{\text {sys }}$ and an exponential cutoff at $E_{\mathrm{c}}=3.1(+0.5-0.4)_{\text {stat }} \pm 0.9_{\text {sys }} \mathrm{TeV}$ and an average integral flux above $2 \mathrm{TeV}$ of $3 \mathrm{Crab}$ flux units. Significant variations of the spectral shape are detected with a spectral hardening as the flux increases. Contemporaneous multi-wavelength observations at lower energies (X-rays and gamma-rays above $\approx 300 \mathrm{GeV}$ ) indicate smaller relative variability amplitudes than seen above $2 \mathrm{TeV}$ during high flux state observed in April 2004.

Key words. galaxies: individual: Mkn 421

\section{Introduction}

Mkn 421 is a "BL Lac" type active galactic nucleus. The broadband spectral energy distribution is dominated by non-thermal emission that is believed to be produced in a relativistic jet pointing towards the observer. The high energy emission of this object has been studied by previous observations carried out by northern hemisphere ground based Cherenkov telescopes (Punch et al. 1992; Aharonian et al. 2002; Krennrich et al. 2002). Observations of Mkn 421 from the southern hemisphere at large zenith angles benefit from considerable increase of the collection area at higher energies, which results in a better temporal resolution at high energies and a better sampling of the high energy part of the energy spectrum (Okumura et al. 2002).

Besides the general interest of understanding the physics of the highly relativistic plasma and its interaction with the ambient medium, the proximity of Mkn $421(z=0.031)$ makes it an interesting target to observe the effect of pair-production of gamma-rays with soft (thermal) background photons as part of the extragalactic background light. As the collection area for large zenith angle observations exceeds two square kilometers at energies beyond $10 \mathrm{TeV}$, the observable energy spectrum could eventually be extended beyond $20 \mathrm{TeV}$, which is important to probe the mid-to-far infrared part of the extragalactic background light.

\section{Observations and data analyses}

HESS (Hofmann et al. 2003) is an imaging atmospheric Cherenkov detector dedicated to the ground based observation of gamma-rays at energies above $100 \mathrm{GeV}$. Situated in Namibia $\left(23^{\circ} 16^{\prime} \mathrm{S} 16^{\circ} 30^{\prime} \mathrm{E}\right)$, the full array of four telescopes is operational since December 2003. Each telescope has a mirror area of $107 \mathrm{~m}^{2}$ and is equipped with a camera consisting of 960 photomultiplier tubes (Vincent et al. 2003). The system has a field of view of $5^{\circ}$ and allows to reconstruct the direction of individual showers with a precision of better than $0.1^{\circ}$.

The HESS observations reported here were carried out for typically 1-2 h per night from MJD 53107.8 to MJD 53114.9 (April 12-19, 2004) triggered by an increased level of X-ray emission detected by the All-Sky-Monitor (ASM) onboard the RXTE satellite and increased activity detected by the Whipple Cherenkov telescope (Krawczynski private communication). Online analysis of the HESS data revealed that the source was also active at $\mathrm{TeV}$ energies motivating an extension of the observational campaign for roughly one week. In the beginning of May (MJD 53134.8 corresponding to May 8, 2004), the HESS array participated in a multi-wavelength campaign with overlap with pointed X-ray observations with the RXTE satellite.

The observation mode was employing all four telescopes, pointing with $0.5^{\circ}$ offset in declination with sign alternating from run to run (28 min each run). The runs were selected according to general quality checks such as absolute value of the trigger rate, relative changes in the rate and performance of the cameras (number of operational pixels, homogeneity of the acceptance). In total, $14.7 \mathrm{~h}$ of good quality data were selected for the analyses. The zenith angle of the observations ranges from $60.3^{\circ}$ at culmination to $65.4^{\circ}$ with an average energy threshold at $1.5 \mathrm{TeV}$. The average size of images and amplitude detected in individual pixels at the given zenith angles is well below the saturation limit of the HESS cameras and read out electronics.

The data were calibrated following the general procedure (Aharonian et al. 2004a) and standard data reduction (image cleaning, event reconstruction) was applied (Aharonian et al. 2005). The cuts used for background rejection were identical to the ones used in previous analyses (e.g. Aharonian et al. 2004b, $2005)$ with the exception of a loose angular cut $\theta^{2}<0.05\left(^{\circ}\right)^{2}$ (compared to $\theta^{2}<0.02\left(^{\circ}\right)^{2}$ at smaller zenith angles), where $\theta^{2}$ is the squared angular distance between source and reconstructed shower direction. The relaxed angular cut compensates for the degrading angular resolution at low elevations. Its value was not optimised to result in a maximum significance but rather chosen to ensure a high $(>80 \%)$ and constant acceptance for gamma-rays. The relative energy resolution at the observed zenith angle range expected from simulation is roughly $25 \%$.

For estimating the background, five off regions identical to the $o n$ region in size and separation from the camera center were chosen (Aharonian et al. 2001). The overall signal detected from Mkn 421 amounts to $N_{\text {on }}=8978$ and $\left\langle N_{\text {off }}\right\rangle=$ 1357.6 averaged over the five background regions with a significance of $S=114 \sigma$ using the likelihood ratio method derived in Li \& Ma (1983).

Finally, in order to reconstruct the energy spectra, collection areas were calculated from simulated air showers taking the range of observed zenith angles and the offset of the source to the camera centre into account. The collection area derived for individual events (using linear interpolation between logarithmic energy bins and linear interpolation between zenith 
angles) were used as weights to calculate the flux in bins of energy by summing over the events in the on and off regions. The effect of spill-over between adjacent energy bins due to the instrument's energy resolution was compensated by using collection areas as a function of reconstructed energy (Mohanty et al. 1998; Aharonian et al. 2002).

Estimates of systematic uncertainties were derived by changing the atmospheric transparency in the simulations and re-applying the analyses to the data. The small variations observed in the cosmic ray induced event rate during the observations (see also next section) are consistent with smaller variations of the atmospheric transparency than assumed in the evaluation of the systematic uncertainties.

\section{Energy spectrum from the Crab nebula at large zenith angles}

In order to check the procedure for the reconstruction of energy spectra at large zenith angles, a dedicated data-set taken on the Crab nebula for $1.8 \mathrm{~h}$ at zenith angles ranging from $57^{\circ}$ to $67^{\circ}$ was analysed.

The fit of a power-law $\left(\mathrm{d} N / \mathrm{d} E=N_{0}(E / \mathrm{TeV})^{-\Gamma}\right)$ to the resulting energy spectrum of the Crab nebula yields $N_{0}=(2.9 \pm$ $\left.0.4_{\text {stat }} \pm 0.7_{\text {sys }}\right) \times 10^{-11} \mathrm{ph} /\left(\mathrm{cm}^{2} \mathrm{~s} \mathrm{TeV}\right), \Gamma=2.6 \pm 0.1_{\text {stat }} \pm 0.3_{\text {sys }}$, $\chi^{2}=7$ (8 d.o.f.). The result is in good agreement with the recently published values given by the HEGRA collaboration (Aharonian et al. 2004c). This gives confidence in the reliability of the instrument's response and reconstruction technique at large zenith angles.

\section{Energy spectra and light-curve from Mkn 421}

The differential energy spectrum for Mkn 421 was calculated in the same way as described above for the Crab nebula. The entire data-set was used to derive a differential energy spectrum.

The final result for the energy spectrum of Mkn 421 is shown in Fig. 1. As the energy spectrum shows clear evidence for curvature, the collection areas were calculated iteratively until the parameters of the model fit function converged. The differential energy spectrum is well sampled between 1 and $15 \mathrm{TeV}$. Between 15 and $30 \mathrm{TeV}$, a signal (corrected for an expected spill-over of 3 events) of $2.2 \sigma$ with $N_{\text {on }}=31$ and $\left\langle N_{\text {off }}\right\rangle=96 / 5=19.2$ events is seen. The collection area for this energy bin exceeds $2 \mathrm{~km}^{2}$ after cuts. Between 30 and $50 \mathrm{TeV}$ an upper limit with a confidence level of $99 \%$ using the method of Feldman \& Cousins (1998) was calculated. All energy bins are centred on the expected average energy for a given bin assuming a spectrum following a power-law with an exponential cutoff.

The result of a fit to the spectrum assuming a power-law with an exponential cutoff (see solid line in Fig. 1), results in $N_{0}=\left(1.55 \pm 0.08_{\text {stat }} \pm 0.4_{\text {sys }}\right) \times 10^{-10} \mathrm{ph} /\left(\mathrm{cm}^{2} \mathrm{~s} \mathrm{TeV}\right)$, $\Gamma=2.1 \pm 0.1_{\text {stat }} \pm 0.3_{\text {sys }}, E_{\mathrm{c}}=3.1(+0.5-0.4)_{\text {stat }} \pm 0.9_{\text {sys }} \mathrm{TeV}$, and $\chi^{2}=16$ (10 d.o.f.). Other model fits using more complicated functions which have been commonly used in the past to describe a curved spectrum were tested: a parabola in $\log (\mathrm{d} N / \mathrm{d} E)$ vs. $\log (E)$ gives $\chi^{2}=12.6$ (9 d.o.f.) and a super-exponential cutoff $\chi^{2}=11.7$ (9d.o.f). The super-exponential cutoff fit

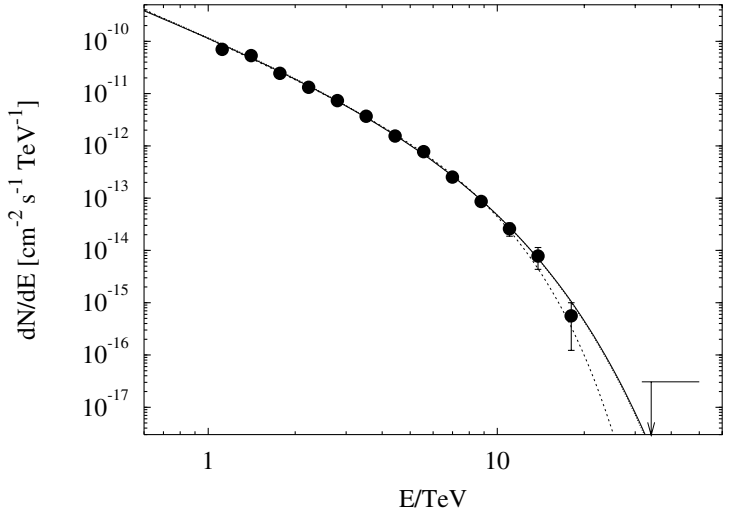

Fig. 1. Differential energy spectrum of Mkn 421: the curvature is described by a power-law with a photon index of $\Gamma=2.1 \pm 0.1_{\text {stat }} \pm$ $0.3_{\text {sys }}$ with an exponential cutoff at $E_{\mathrm{c}}=3.1(+0.5-0.4)_{\text {stat }} \pm$ $0.9_{\text {sys }} \mathrm{TeV}$ (solid line) or alternatively with a super-exponential cutoff $\left(\exp \left(-\left(E / E_{\mathrm{c}}\right)^{1.6 \pm 0.3}\right)\right.$ at $E_{\mathrm{c}}=\left(6.25 \pm 0.4_{\text {stat }} \pm 0.9_{\text {sys }}\right) \mathrm{TeV}$ (dashed line). Between 30 and $50 \mathrm{TeV}$ an upper limit with a $99 \%$ confidence level is displayed.

with $\mathrm{d} N / \mathrm{d} E \propto E^{-\Gamma} \times \exp \left(-\left(E / E_{\mathrm{c}}\right)^{\alpha}\right)$ with $\alpha=1.6 \pm 0.3$ (systematic uncertainty negligible) is included in Fig. 1 as a dashed line. According to the F-test, the probability of a chance improvement of the $\chi^{2}$ for the super-exponential cutoff is $6.4 \%$ which is not sufficiently low to give a preference to this fit function (the same holds true for the parabolic fit function).

The cutoff energy $E_{\mathrm{c}}=3.1(+0.5-0.4)_{\text {stat }} \pm 0.9_{\text {sys }} \mathrm{TeV}$ found in the observations described here is fully consistent with previous observations in the years 2000 and 2001 by HEGRA of 3.6 $(+0.4-0.3)_{\text {stat }}(+0.9-0.8)_{\text {sys }} \mathrm{TeV}$ (Aharonian et al. 2002) and VERITAS (4.3 \pm 0.3$) \mathrm{TeV}$ (Krennrich et al. 2002).

The diurnal integral fluxes above $2 \mathrm{TeV}$ were calculated by taking the sum over the inverse collection area for the events with energies above the chosen value. With the threshold energy raised to $2 \mathrm{TeV}$, this gives an estimate of the flux which is independent of the variation of the actual energy threshold within a night, at the expense of loosing count statistics. As an additional benefit, the dependence on changes of the spectral shape are negligible as the collection area is not strongly changing with energy for energies well above the threshold. The resulting light-curve is shown in Fig. 2.

The light-curve exhibits night-by-night variability in the first observing week and resumes a lower flux in the later observation. The peak diurnal average flux reaches a value of about 5 times the flux observed from the Crab nebula. The corresponding gamma-ray rate is sufficiently high to probe intranight variability of the high energy end of the spectrum with unprecedented accuracy.

During the observations of MJD 53113.8-53113.9 (April 18) and 53114.8-53114.9 (April 19), significant variations of the flux within these nights are detected. The hypothesis of a constant flux during these nights results in $\chi^{2}=$ 40.6(7 d.o.f.) and $\chi^{2}=28$ (7 d.o.f.) respectively. The intra-night variations as seen during MJD 53113.8-53113.9 (April 18) using bins of 14 min width are shown as an inlay in Fig. 2. In order to exclude variations of the detector's response or changes in the atmosphere to be responsible for the observed variations, 


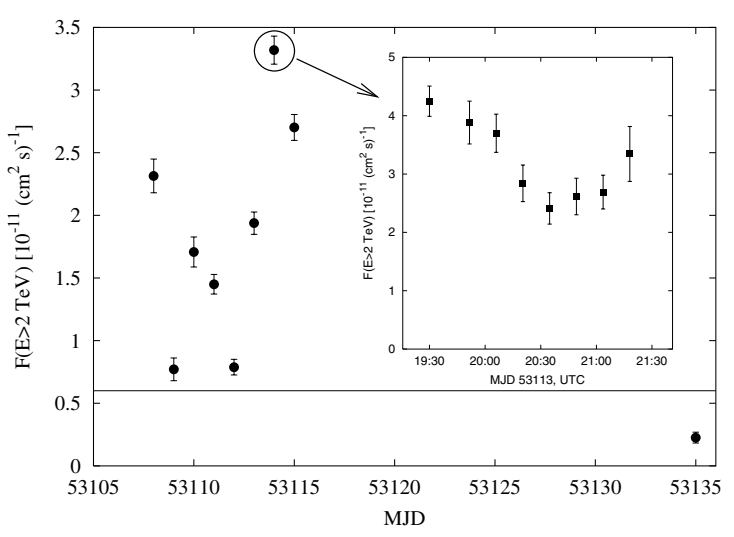

Fig. 2. The light-curve for Mkn 421 in April and May with diurnal integral flux values above $2 \mathrm{TeV}$. For comparison, the horizontal line indicates the flux observed from the Crab nebula. The inlay shows the light-curve for the night of MJD 53113 (marked with a circle and an arrow) with the strongest indication for intra-night variability in 14 min bins $(0.01 \mathrm{~d})$.

the post-cut (after applying the image shape cuts) cosmic ray rate has been checked for variability which is smaller than $2 \%$ in relative root mean square (rms) during this night. The observed significant intra-night variability in the light-curve suggests a decay time of less than $1 \mathrm{~h}$.

The light-curve is not corrected for possible variations of the atmospheric transparency. The atmospheric transmissivity between the position of the air shower and the detector can be probed by the rate of detected cosmic ray events, as the Cherenkov light traverses the same aerosol layer which is believed to dominate possible temporal variations of the atmosphere's transparency. During the observations, the average cosmic ray rates for individual runs varied between 113 and $143 \mathrm{~Hz}$ with an average of $132 \mathrm{~Hz}$ and rms of $13 \mathrm{~Hz}$. The post-cut rate shows even less variation with $8 \%$ relative rms. The observed variability of Mkn 421 is therefore clearly associated with the source and not a consequence of temporal variations of the detector response or atmospheric transparency. In principle, a correction of the measured gamma-ray flux could be derived and applied to the light-curve. This was not done as the effect is small $(<10 \%)$ in comparison to the observed variability.

In order to study variations of the spectral shape in various flux states, diurnal spectra were calculated. Given the correlation between the cutoff energy $E_{\mathrm{c}}$ and the photon index $\Gamma$ derived from the fit of a power-law with an exponential cutoff (correlation coefficient -0.955 between $E_{\mathrm{c}}^{-1}$ and photon in$\operatorname{dex} \Gamma)$, the power-law index was kept constant $(\Gamma=2)$ for the fits applied to spectra obtained for individual nights. The fit was applied to a fixed energy range from 1 to $10 \mathrm{TeV}$. As a result, a hardening of the spectrum or an increase of the cutoff energy is seen clearly in the correlation of the cutoff energy $E_{\mathrm{c}}$ and the integral flux above $2 \mathrm{TeV}$ in Fig. 3. A similar result was obtained when keeping the cutoff energy fixed and letting the photon index vary freely.

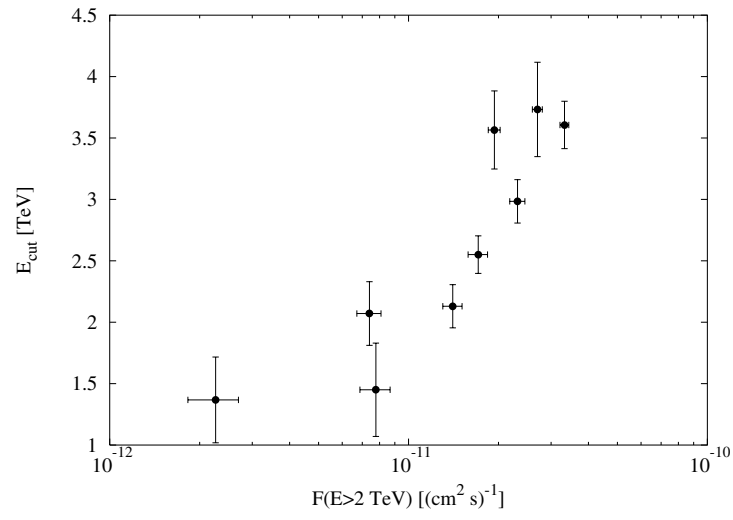

Fig. 3. The cutoff energy as determined from a fit with a fixed powerlaw photon index $\Gamma=2$ versus integrated flux measured for $E>$ $2 \mathrm{TeV}$. Note, that the point with the lowest flux is from the May observations.

\section{Multiwavelength observations in April 2004}

During April 2004, a coordinated multi-wavelength campaign monitored the activity of Mkn 421 in radio, optical, X-ray, and gamma-rays (Cui et al. 2004). The source was seen to be active in X-rays where observations with the array of proportional chamber units (PCU) onboard the RXTE satellite were performed. These observations were not simultaneous with the observations with the HESS array, but by combining the PCU with the ASM data a good temporal coverage overlapping with the HESS observations can be achieved. An average of 4 counts/s were detected by the ASM during the first weeks of April. This is sufficiently high to probe the activity of Mkn 421 during individual ASM pointings (dwells).

The Whipple $10 \mathrm{~m}$ Cherenkov telescope was observing Mkn 421 simultaneously with the RXTE pointings which were generally starting within a few hours after the HESS observations took place. First preliminary results of this campaign have been presented by Cui et al. (2004).

In Fig. 4, the different observations are combined such that the observed flux (or count-rate) is normalized to the average flux (or count-rate) during the time between MJD 53107 and 53116 . The preliminary Whipple light-curve is derived from the count-rate which is not corrected for different zenith angles of observations. Generally, the Whipple observations were carried out at small zenith angles (Cui et al. 2004) and according to previous observations, the energy threshold is estimated to be $\approx 400 \mathrm{GeV}$ (Krennrich et al. 2002). The ASM light-curve and the $1 \sigma$ uncertainty band is obtained by calculating the sliding average over five dwells.

Even though a detailed correlation analysis is difficult because of a large fraction of the data not being simultaneous, it is interesting to note that the strongest variability occurs at the highest energies, with a maximum relative amplitude of the observed flux of $F_{\max } / F_{\min }=4.3 \pm 0.5$ as compared to $1.7 \pm 0.2$ derived from the Whipple count rates and $2.05 \pm 0.02$ from the RXTE PCU observations. As a measure of the variability the relative rms of the measurement indicates a variability 


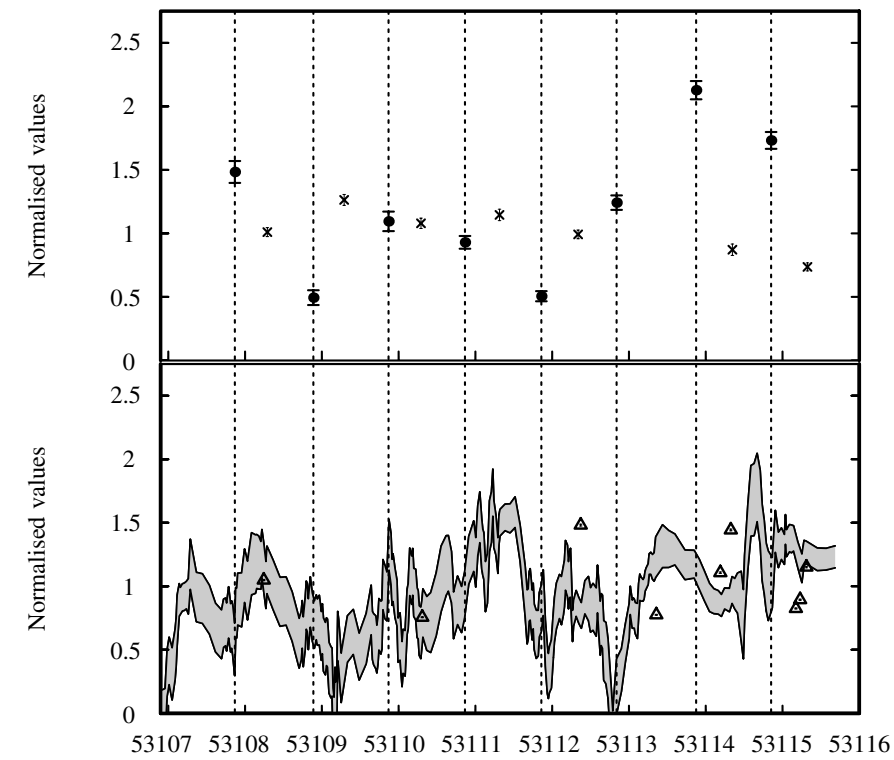

Fig. 4. The relative changes of the flux with respect to the average observed during the multi-wavelength campaign in April 2004. Upper panel: HESS $\mathrm{F}(E>2 \mathrm{TeV})$ (filled circles), Whipple (crosses, preliminary count rate without correction for varying zenith angles). Lower panel: PCU (open triangles, count-rate), ASM ( $1 \sigma$ error band, see text for details). Whipple and RXTE PCU data taken from Cui et al. (2004).

of $(17 \pm 6) \%$ seen by the Whipple instrument and $(26 \pm 1) \%$ for the X-ray data taken with the PCU detectors, whereas for HESS the relative rms value is $(51 \pm 8) \%$.

\section{Conclusion}

The results on the time averaged energy spectrum of Mkn 421 presented here confirm previous observations of the existence of a cutoff at $E_{\mathrm{c}}=3.1(+0.5-0.4)_{\text {stat }} \pm 0.9_{\text {sys }} \mathrm{TeV}$ which is lower than the cutoff energy of $6.2 \pm 0.4_{\text {stat }}(+2.9-1.5)_{\text {sys }} \mathrm{TeV}$ observed from Mkn 501 in 1997 (Aharonian et al. 1999). Given the similar red shift of these two objects, this would imply that the cutoff in the Mkn 421 spectrum is intrinsic to the source and not due to absorption. With the observations carried out at large zenith angles and increased collection area, a signal at the level of $2.2 \sigma$ (corrected for the effect of spill-over) was detected between 15 and $30 \mathrm{TeV}$. This detection in conjunction with the observation of a cutoff in the energy spectrum intrinsic to the source is important for constraining the extragalactic background light at wavelengths beyond $10 \mu \mathrm{m}$. Furthermore, the energy spectrum was observed to become harder as the integral flux increases. It is not possible to discern whether the hardening is a consequence of an increase of the cutoff energy from 1.5 to $3.5 \mathrm{TeV}$ or a change in the power-law index as these two parameters are highly correlated.

The relative amplitude and variance of the variability observed above $2 \mathrm{TeV}$ is significantly higher than observed at lower energies during the same activity period by the Whipple telescope and the RXTE pointed X-ray detector. The sparse but similar sampling of the light curve among the different observations (HESS, Whipple, and RXTE PCU) does not introduce a bias which could explain the observed difference in the variability.

The larger variability seen at multi-TeV energies compared to energies below $\mathrm{TeV}$ is consistent with the observed spectral changes, where the relative increase of the flux at higher energies causes a hardening of the observed spectra. Assuming the correlation between integral flux and cut-off energy indicated in Fig. 3 and using the integral flux above $2 \mathrm{TeV}$ as measured with HESS, the expected light curve above $400 \mathrm{GeV}$ was calculated. As expected, the relative rms scatter of the lower energy light curve is roughly half of the value observed above $2 \mathrm{TeV}$ well consistent with the observed variability in the Whipple light curve.

Acknowledgements. The support of the Namibian authorities and of the University of Namibia in facilitating the construction and operation of HESS is gratefully acknowledged, as is the support by the German Ministry for Education and Research (BMBF), the Max Planck Society, the French Ministry for Research, the CNRS-IN2P3 and the Astroparticle Interdisciplinary Programme of the CNRS, the U.K. Particle Physics and Astronomy Research Council (PPARC), the IPNP of the Charles University, the South African Department of Science and Technology and National Research Foundation, and by the University of Namibia. We appreciate the excellent work of the technical support staff in Berlin, Durham, Hamburg, Heidelberg, Palaiseau, Paris, Saclay, and in Namibia in the construction and operation of the equipment.

\section{References}

Aharonian, F. A., Ahkperjanian, A. G., Barrio, J. A., et al. (HEGRA coll.) 1999, A\&A, 349, 11

Aharonian, F., Akhperjanian, A., Barrio, J. A., et al. (HEGRA coll.) 2001, A\&A, 370, 112

Aharonian, F., Akhperjanian, A., Beilicke, M., et al. (HEGRA coll.) 2002, A\&A, 393, 89

Aharonian, F., Akhperjanian, A. G., Aye, K.-M., et al. (HESS coll.) 2004a, Astroparticle Physics, 22, 109

Aharonian, F., Akhperjanian, A. G., Aye, K.-M., et al. (HESS coll.) 2004b, A\&A, 425, L13

Aharonian, F., Akhperjanian, A., Beilicke, M., et al. (HEGRA coll.) 2004c, ApJ, 614, 897

Aharonian, F., Akhperjanian, A. G., Aye, K.-M., et al. (HESS coll.) 2005, A\&A, 430, 865

Cui, W., Blazejowski, M., Aller, M., et al. 2005, Proc. of the Heidelberg Symposium on High Energy Gamma Ray Astronomy, ed. F. A. Aharonian, H. J. Völk, \& D. Horns, AIP Conf. Proc. Ser., 745,455

Feldman, G. J., \& Cousins, R. D. 1998, Phys. Rev. D, 57, 3873

Hofmann, W. 2003, Proc. of 28th ICRC, ed. T. Kajita, Y. Asaoka, A. Kawachi, Y. Matsabura, \& M. Sasaki (Universal Academy Press), 3,2811

Krennrich, F., Bond, I. H., Bradbury, S. M., et al. 2002, ApJ, 575, L9

Li, T.-P., \& Ma, Y.-Q. 1983, ApJ, 272, 317

Mohanty, G., Carter-Lewis, D. A., Fegan, D. J., et al. 1998, Astroparticle Physics, 9, 15

Okumura, K., Asahara, A., Bicknell, G. V., et al. 2002, ApJ, 579, L9

Punch, M., Akerlof, C. W., Cawley, M. F., et al. 1992, Nature, 358, 477

Vincent, P., Denance, J.-P., Huppert, J.-F., et al. 2003, Proc. of 28th ICRC, ed. T. Kajita, Y. Asaoka, A. Kawachi, Y. Matsabura, \& M. Sasaki (Universal Academy Press), 3, 2887 